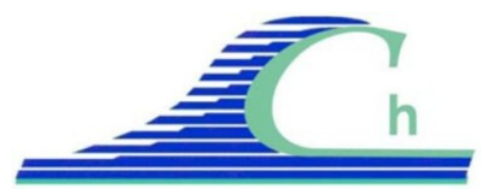

XII ${ }^{\text {èmes }}$ Journées Nationales Génie Côtier - Génie Civil

Cherbourg, 12-14 juin 2012

DOI:10.5150/jngcgc.2012.065-M C Editions Paralia CFL

disponible en ligne - http://www.paralia.fr - available online

\title{
Bathymétrie de l'embouchure du bassin d'Arcachon par fusion de données hétéroclites et reconstruction bathymétrique
}

\author{
Vincent MARIEU ${ }^{1}$, Thomas GUERIN ${ }^{2}$, Sylvain CAPO ${ }^{1}$, Driss BRU ${ }^{1}$, \\ Bertrand LUBAC ${ }^{1}$, Vincent HANQUIEZ ${ }^{1}$, \\ Virginie LAFON $^{1}$, Philippe BONNETON ${ }^{1}$
}

1. Université de Bordeaux, UMR CNRS 5805 EPOC, Avenue des facultés, 33405 Talence, France.

v.marieu@epoc.u-bordeaux1.fr

2. ARTELIA Maritime, Le Rubis, 10 rue Gutenberg, 33697 Mérignac Cedex, France.

Thomas.GUERIN@arteliagroup.com

\section{Résumé :}

Cet article présente les premiers résultats de l'application de nouvelles méthodes pour reconstruire la bathymétrie d'une zone à partir de données multi-sources. La zone d'étude concernée ici est l'embouchure du bassin d'Arcachon, zone très dynamique, siège à la fois de houles énergétiques et de courants de marée intenses. Les données sont issues de sondages bathymétriques mais sont également extraites d’images satellitaires optiques, par l'application de diverses techniques d'inversion. Une méthode d'interpolation des données a été développée spécifiquement pour cette application, consistant en une méthode de krigeage itérative tenant compte de l'anisotropie locale en chaque point, de manière à retrouver au mieux la forme des structures sédimentaires. Des données fictives doivent être reconstruites pour éviter les trous de données avant d'effectuer l'interpolation. Alliée à la méthode d'interpolation, cette reconstruction de données permet d'obtenir des bathymétries complètes du bassin d'Arcachon.

Mots-clés :

Bathymétrie - Embouchure - Télédétection - Optique marine - Sondages bathymétriques - Interpolation - Krigeage anisotrope - Reconstruction de données

\section{Introduction}

L'embouchure du bassin d'Arcachon est un environnement très dynamique, soumis à la fois à un régime méso/macro-tidal et à une houle oblique énergétique (CAYOCCA, 1996). La zone des passes, montrée figure 1 , s'étend sur une surface d'environ 50 km² et sa morphologie évolue très rapidement, ce qui nécessite d'effectuer des sondages bathymétriques réguliers pour assurer un balisage efficace des chenaux maritimes. Les difficultés d'accès à la zone pour des navires bathymétriques ainsi que l'importante surface à parcourir rendent cette tâche longue et coûteuse, justifiant le développement de techniques basées sur l'imagerie satellitaire pour effectuer le suivi morphologique de 
l'embouchure. Ce travail est effectué par le laboratoire EPOC de l'université de Bordeaux depuis plusieurs années en collaboration avec le Syndicat Intercommunal du Bassin d'Arcachon (FROIDEFOND \& LAFON, 2009), à l'aide de la base de données KALIDEOS-Littoral (http://kalideos.cnes.fr) regroupant des images optique SPOT et FORMOSAT. Ce suivi, essentiellement qualitatif, est cependant insuffisant pour obtenir des modèles numériques de terrain complets de l'embouchure.

Afin d'obtenir des bathymétries complètes de la zone, les sondages bathymétriques effectués sur la zone ont été complétés par un maximum de données provenant de l'imagerie satellitaire. L'interpolation des données a été effectuée avec une méthode de krigeage adaptative prenant en compte l'anisotropie locale des données, adaptée à la géométrie complexe de l'embouchure et à la répartition très hétérogène des données. Dans un deuxième temps, il est nécessaire de combler les zones sans données. Cette partie du travail est actuellement en cours de développement, la morphologie et le déplacement des structures sédimentaires telles que barres ou chenaux sont étudiés pour permettre de reconstruire les données manquantes et d'obtenir des bathymétries complètes de la zone. Les premiers résultats de ce travail sont présentés dans les paragraphes suivants pour le mois de juin 2009, riche en données de sources variées.
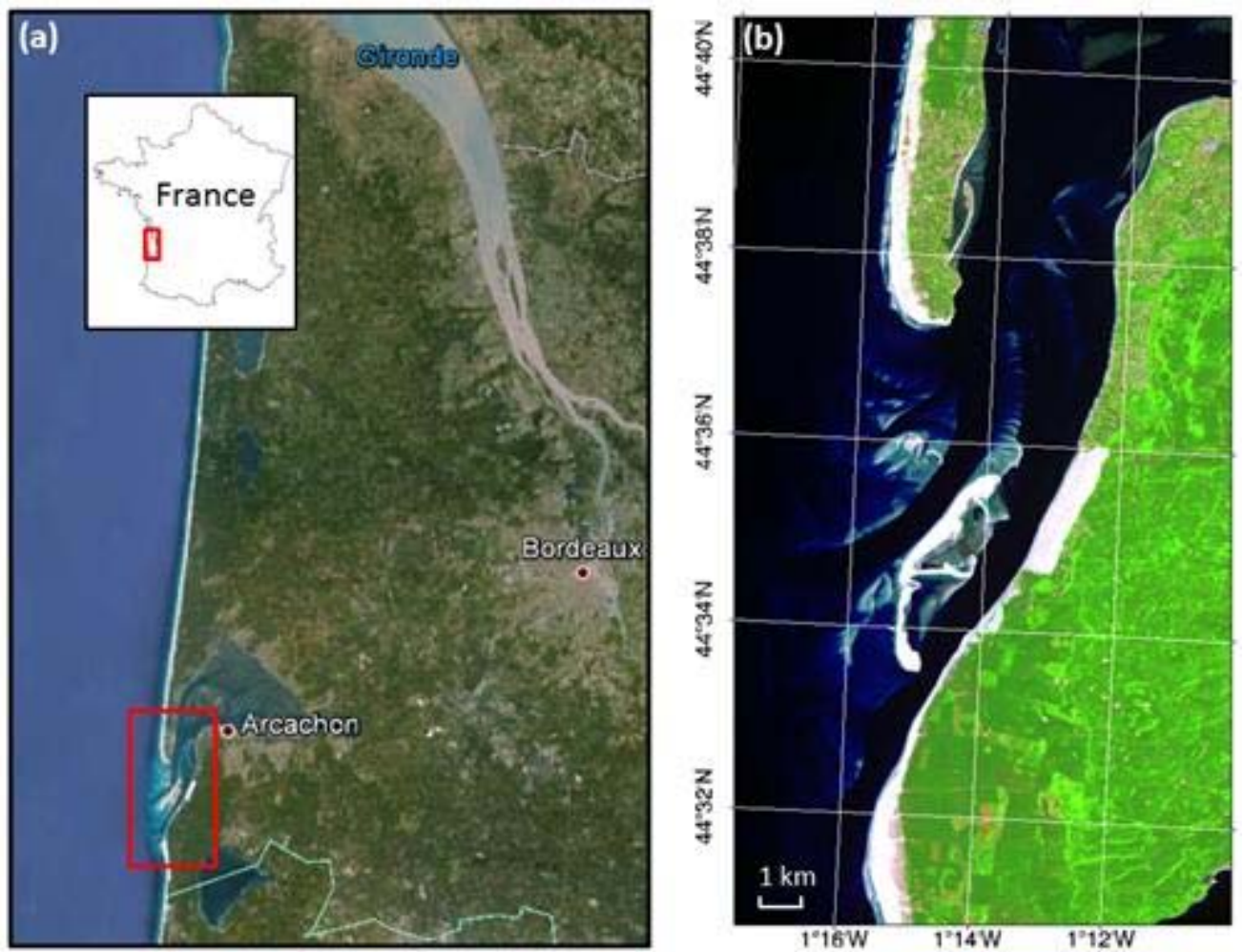

Figure 1. Situation géographique du bassin d'Arcachon (a) et Scène SPOT-5@CNES montrant l'embouchure du bassin le 24 juin 2009 (b), recomposition colorée à partir des bandes verte (XS1), proche infrarouge (PIR) et moyen infrarouge (MIR). 


\section{XII ${ }^{\text {èmes }}$ Journées Nationales Génie Côtier - Génie Civil \\ Cherbourg, 12-14 juin 2012}

\section{Recherche et compilation des données}

\subsection{Sondages bathymétriques}

Les sondages bathymétriques sont réalisés par un navire bathymétrique équipé d’un echo-sondeur acoustique monofaisceau et d'un GPS différentiel dans la zone des passes du bassin d'Arcachon, sous la direction du Syndicat Intercommunal du Bassin d'Arcachon (SIBA). Le but de ces campagnes de sondages est de garder le balisage des chenaux de navigation à jour. Un objectif du SIBA est d'obtenir une couverture complète de la zone une fois par an, au minimum, ces données étant réalisées régulièrement depuis 2000. La précision requise pour le balisage des chenaux rend ces campagnes très longues, environ une journée est nécessaire pour balayer la moitié d’une passe. De plus, des conditions de houle calme et de marée montante sont nécessaires pour l'acquisition, autant de contraintes qui ne permettent pas l'obtention d'une bathymétrie complète de la zone en quelques jours. Les données sont donc réparties par zones sur toute l'année. La trajectoire du bateau sondeur suit des transects perpendiculaires aux chenaux espacés de 50 à 200 mètres. Les données de sondage sont très denses suivant la trajectoire du bateau ( $\sim 20 \mathrm{~cm}$ de résolution) et peuvent être souséchantillonnées pour éviter un déséquilibre avec la densité des pixels des images satellitaires (20 m pour SPOT-4, 10 m pour SPOT-5). En plus des données elles-mêmes, les sondages bathymétriques peuvent être utilisés pour calibrer les équations d’inversion des données satellites.

\subsection{Inversion des données de réflectance}

Le but de ce papier n'est pas de développer un algorithme complexe d'inversion des données satellites, ce travail étant effectué par ailleurs dans le cadre du projet INFOLITTORAL (http://infolittoral.spotimage.com/). L'algorithme utilisé ici est le plus simple possible, basé sur la loi de Beer-Lambert (LYZENGA, 1978) décrivant une atténuation exponentielle de la lumière en fonction de la profondeur, $z$, pour une longueur d'onde donnée. Les données de sondage du mois de juin 2009 sont utilisées pour calculer les coefficients correspondant à l'inversion de la scène SPOT-5 du 24 juin 2009. Nous avons utilisé un algorithme d'inversion précédemment appliqué à cette zone par LAFON et al. (2002). Cet algorithme est basé sur une seule bande, la bande verte de SPOT (XS1), qui présente la meilleure pénétration dans l'eau. Il est simple mais robuste lorsque des données de sondage sont disponibles. L'algorithme n’utilisant pas de réflectances au sol, le compte numérique dans la bande XS1, c'est-à-dire la donnée brute non calibrée du capteur, peut être utilisé directement. L'heure de la prise de vue est utilisée pour ajouter à la bathymétrie la hauteur d'eau mesurée à Arcachon, au marégraphe de la jetée d'Eyrac. La figure 2 montre la correspondance entre les données de sondages moyennées par pixel et le compte numérique correspondant dans la bande XS1. Les coefficients de l'équation d'inversion (trait plein) sont dérivés des données par 
une approximation des moindres carrés, et un seuil de $4 \mathrm{~m}$ (trait pointillé) est fixé arbitrairement pour limiter l'erreur d'inversion dans les plus grandes profondeurs.

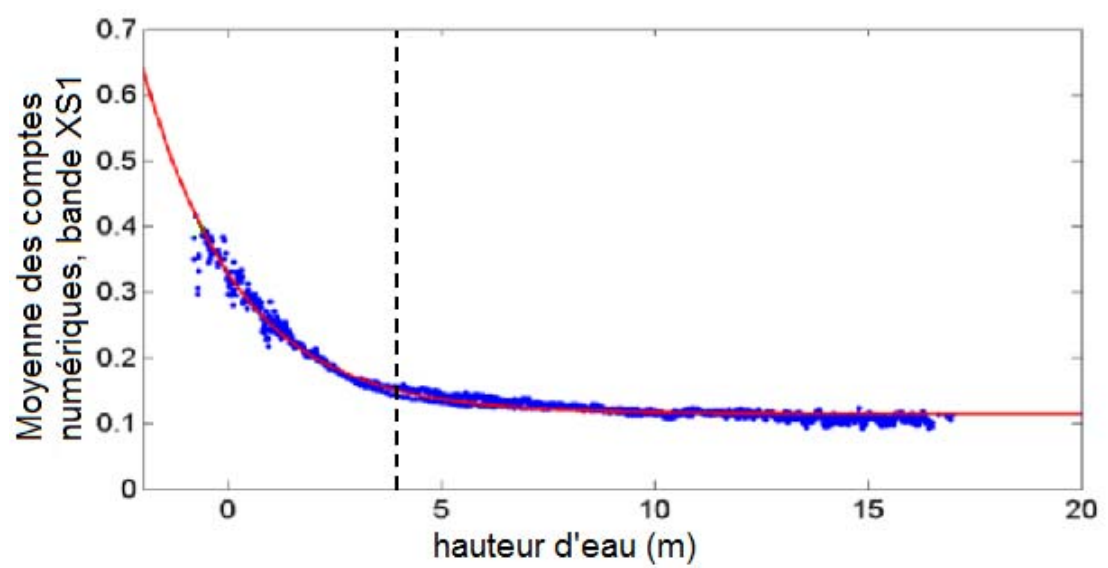

Figure 2. Comptes numériques normalisés de la bande XS1 pour la scène SPOT-5 du 24 juin 2009 en fonction de la hauteur d'eau moyennée par pixels, mesurée lors des sondages réalisés durant le mois de juin 2009 (points), courbe de régression exponentielle utilisée pour l'inversion (trait plein) et seuil à $4 \mathrm{~m}$ (trait pointillé)

2.3 Inversion de l'équation de dispersion des vagues à partir de la détection de crêtes de houle

Sur certaines scènes satellites les crêtes de houle sont facilement détectables au large de l'embouchure, suivant la longueur d'onde de la houle, sa hauteur et l'angle d'inclinaison de la lumière du soleil. Ces crêtes ont été utilisées pour recalculer la hauteur d'eau au large de l'embouchure, en association avec la mesure de la période de la houle réalisée par la bouée houle du Cap Ferret disponible au Centre d'Archivage National de Données de Houle In Situ (CANDHIS). La méthode utilisée ici est relativement simple et dérivée de LEU \& CHANG (2005). L'inversion de l'équation de dispersion de la houle nous permet de calculer la hauteur d'eau $h$, en fonction de la longueur d'onde des vagues $\lambda$, de leur période $T$ et de la constante de gravité $g$ :

$h=\frac{\lambda}{2 \pi} \operatorname{atanh}\left(\frac{2 \pi \lambda}{g T^{2}}\right)$

La période pic des vagues est donnée par la bouée houle et la longueur d’onde pic est calculée sur des sous-images de la bande proche infrarouge (PIR) de la scène satellite, le signal étant le plus net dans cette bande du fait de la faible pénétration de l’infra-rouge dans l'eau. La figure 3 montre les étapes de détection de la longueur d'onde pic pour une sous-image (figure 3.a). Le spectre d'énergie de la transformée de Fourier 2D de la sous-image est calculé (figure 3.b), puis ce spectre est sommé sur un angle de $360^{\circ}$ de manière à obtenir la densité spectrale d'énergie de la sous-image en fonction du nombre d'onde, quelle que soit la direction de la houle. La méthode est sensible à la profondeur 


\section{XII èmes Journées Nationales Génie Côtier-Génie Civil \\ Cherbourg, 12-14 juin 2012}

d'eau relative au point calculé, cet aspect étant discuté par LEU \& CHANG (2005) et, dans notre cas, la méthode est nettement moins précise que les autres méthodes utilisées. Elle est cependant appliquée pour couvrir la bathymétrie au large de l'embouchure, où la profondeur importante ne permet pas d'utiliser une autre méthode.
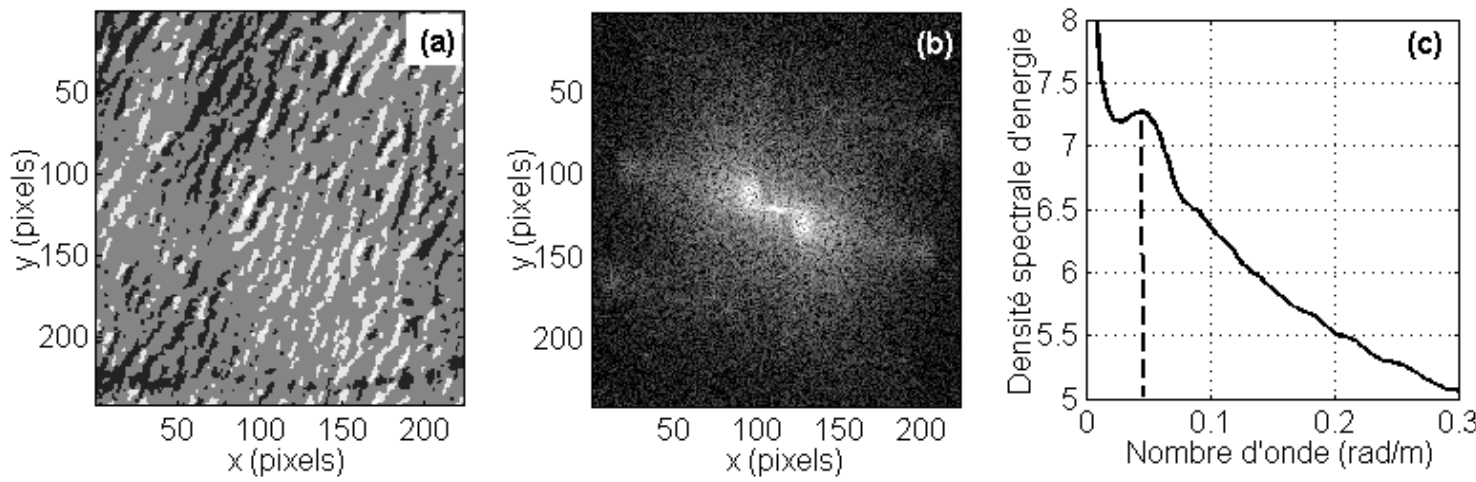

Figure 3. Imagette de la bande PIR (a), module de la transformée de Fourier 2D de l'imagette (b) et spectre moyen sur $360^{\circ}$ (c), la position correspondant à la longueur d'onde de la houle étant repérée d'un trait pointillé rouge.

\section{Interpolation des données par krigeage avec anisotropie locale}

Dans ce travail, un effort particulier a été porté sur la méthode d'interpolation des données. En effet, les données ne couvrent pas complètement la zone et des trous important sont présents. En particulier, certaines parties des chenaux ne sont pas sondées très souvent et les méthodes d'interpolation isotropes ont tendance à "boucher" les chenaux, les points les plus proches du fond des chenaux étant ceux obtenus en bord de chenal par inversion de l'image satellite. En conséquence, il est nécessaire de prendre en compte l'anisotropie de la zone mais celle-ci n'est pas constante sur toute l'embouchure et un calcul local doit être effectué. Nous nous sommes basés sur le travail de TE STROET \& SNEPVANGERS (2005), méthode itérative consistant en un calcul de l'anisotropie locale en chaque point de la grille, puis en un krigeage stationnaire à moyenne inconnue (appelé aussi krigeage ordinaire) avec prise en compte de l'anisotropie locale sur toute la grille. Bien que l'algorithme soit identique, l'angle et le ratio de l'anisotropie n'ont pas été calculés comme TE STROET \& SNEPVANGERS (2005), leur méthode se révélant fausse pour le calcul des ratios. Dans cet article, l'anisotropie locale est calculée sur une sous-fenêtre autour de chaque maille de la grille, à partir des variations de pente dans la sous-fenêtre. La direction montrant la plus grande variation de pente est retenue, ainsi que sa perpendiculaire. Cette dernière direction définit l'axe principal d'anisotropie et le ratio d'anisotropie est donné par le rapport des variations de pente selon ces deux directions. La taille de la sous-fenêtre doit être en relation avec la dimension des structures sédimentaires de la zone ainsi qu'avec la taille des mailles de la grille. Au premier pas de temps, l'interpolation sur la 
grille est effectuée avec un krigeage ordinaire sur les données, sans anisotropie, la grille obtenue est alors utilisée pour calculer l'anisotropie locale en chaque maille. Le krigeage ordinaire avec anisotropie locale est ensuite appliqué sur les données pour calculer une nouvelle grille. Ce procédé est répété jusqu’à ce que les différences entre les grilles successives deviennent peu importantes. La figure 4 montre le résultat après quatre itérations sur une sous-zone du bassin présentant de fortes anisotropies.

Après le krigeage isotrope de la première itération (figure 4.a), les bords de chenaux sont utilisés pour le calcul des points du milieu de chenal, ce qui induit une rupture du chenal. Le résultat est nettement amélioré après la quatrième itération (figure 4.d) la continuité du chenal étant assurée. La taille de la fenêtre glissante de calcul de l'anisotropie locale a été fixée à 1000 mètres, en correspondance avec l'échelle des structures morphologiques présentes dans l'embouchure. TE STROET \& SNEPVANGERS (2005) font diminuer la taille de la fenêtre de calcul locale à chaque itération, de manière à raffiner le calcul de l'anisotropie pour capturer les plus petites échelles morphologiques. Dans notre cas, ce procédé n’a pas donné de résultats probants, certainement du fait de la taille relativement grande des structures sédimentaires (de l'ordre de quelques centaines de mètres).
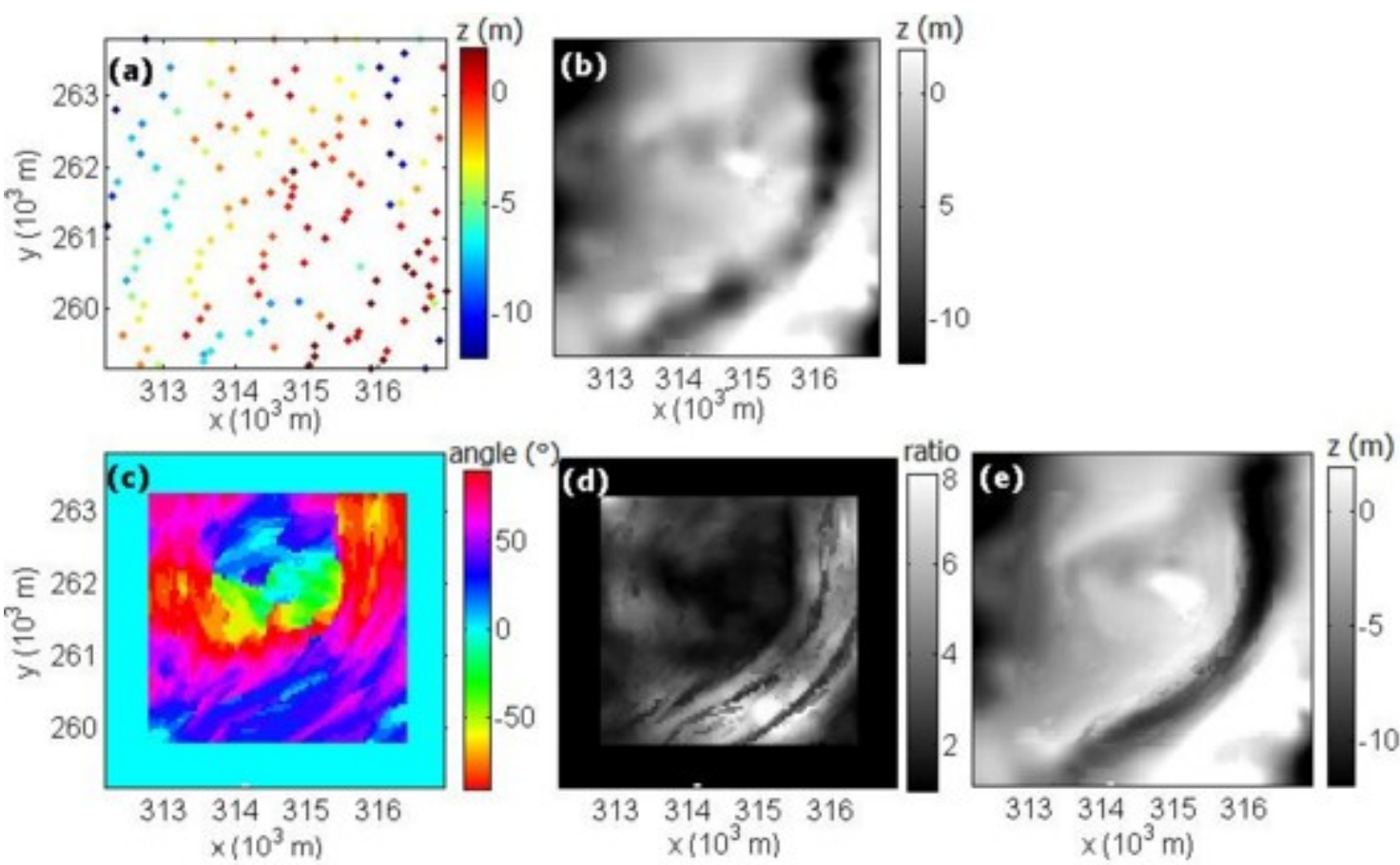

Figure 4. Points de mesure sur une zone test de l'embouchure (a), bathymétrie après la première itération (b), angle (c) et ratio (d) de l'anisotropie après la $3^{\text {ème }}$ itération, et bathymétrie après la $4^{\text {ème }}$ itération (e). 


\section{XII ${ }^{\text {èmes }}$ Journées Nationales Génie Côtier - Génie Civil \\ Cherbourg, 12-14 juin 2012}

\section{Reconstruction de données manquantes}

Dans certaines zones, il manque trop de points de mesures pour espérer une interpolation correcte sur la grille. En juin 2009, c'est en particulier le cas sur la partie interne (nord) de la passe nord, qui n’a pas été sondée, ainsi que pour la partie centrale de la passe sud. Le but de ce travail étant de fournir des bathymétries pour la modélisation numérique des circulations dans l'embouchure, il est crucial de reconstruire ces zones manquantes, même si cela implique des imprécisions sur la bathymétrie obtenue, les modèles ne pouvant fonctionner sur des grilles incomplètes. Cette partie du travail est actuellement en développement et la première phase a été de déterminer la position du centre des chenaux manuellement à partir de la bande PIR (peu de pénétration dans l'eau) de l'image satellite de juin 2009, puis les valeurs de la bathymétrie sur cette ligne ont été extrapolées à partir de sondages effectués les années précédentes en ces points, permettant ainsi de reconstruire des lignes de chenaux. La figure 5.a montre le bilan des données mesurées par les diverses méthodes, ainsi que les portions reconstruites des chenaux. Alliée à la méthode de krigeage ordinaire avec anisotropie locale, ces points permettent de reconstruire une bathymétrie complète et réaliste de l'embouchure du bassin d'Arcachon, comme montré figure 5.b.

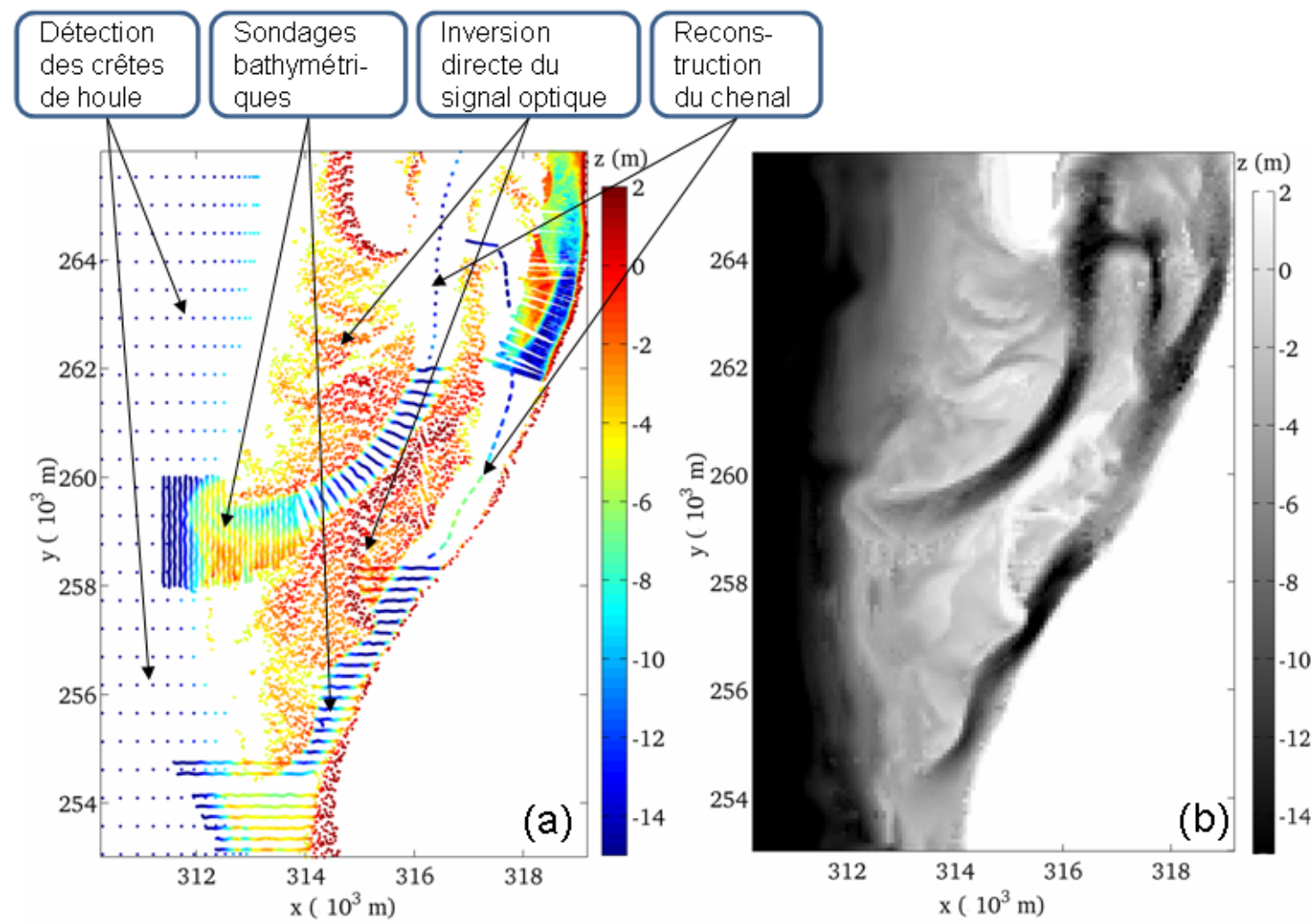

Figure 5. Bilan des données compilées pour le mois de juin 2009 (a) et bathymétrie complète de l'embouchure après interpolation sur une grille constituée de mailles de $75 m(b)$. 


\section{Conclusions}

Ce travail montre un assemblage de méthodes permettant de reconstruire des modèles numériques de terrain sur une zone peu accessible du littoral. En alliant mesures in situ et télédétection satellitaire optique, il est possible de couvrir la totalité des $50 \mathrm{~km}^{2}$ de l'embouchure du bassin d'Arcachon. Chacun des aspects de ce travail peut être amélioré individuellement. C'est en particulier le cas des méthodes d'inversion des images satellitaires, des méthodes étant actuellement à l'étude au sein du laboratoire pour s'affranchir des sondages bathymétriques. La partie concernant la reconstruction de données peut être significativement améliorée, des modèles de fonctionnement des structures sédimentaires doivent être établis à partir des mesures précédentes. Il est intéressant de noter que les bathymétries obtenues permettront elles-mêmes d'améliorer la compréhension du fonctionnement de l'embouchure, fournissant ainsi de meilleurs modèles. Par la suite, il sera également possible de quantifier les incertitudes propres à chacune des méthodes afin d'associer une carte des incertitudes à chacune des bathymétries. Pour ce faire, il est nécessaire de prendre en compte les incertitudes liées à la mesure lors des sondages, celle liée aux différentes méthodes d’inversion bathymétrique, l'incertitude liée à l'interpolation et celle liée à la reconstruction bathymétrique, cette dernière étant très difficile à évaluer du fait qu'elle intègre toutes les précédentes. Les perspectives de ce travail sont variées. Dans un premier temps, les bathymétries obtenues serviront de base à la simulation numérique de l'hydrodynamique et éventuellement de la morphodynamique de l'embouchure. Dans un second temps, ce travail pourra avoir des applications pour la gestion du balisage de l'embouchure, les sondages pouvant être effectués en priorité dans les zones à forte incertitude ou les plus en mouvement.

\section{Références bibliographiques}

CAYOCCA F. (1996). Modélisation morphodynamique d'une embouchure tidale : Application aux passes d'entrée du Bassin d'Arcachon. Thèse, Université Bordeaux 1. FROIDEFOND J.-M., LAFON V. (2009). Surveillance des passes du bassin d'Arcachon en 2009 par imagerie SPOT-5. Rapport final SIBA 2009, U. Bordeaux 1.

LAFON V., FROIDEFOND J.-M., LAHET F., CASTAING P. (2002). SPOT shallow water bathymetry of a moderately turbid tidal inlet based on field measurements. Remote Sensing of Environment, $n^{\circ}$ 81, pp 136-148. doi:10.1016/S0034-4257(01)00340-6 LEU L.-G., CHANG H.-W. (2005). Remotely sensing in detecting the water depths and bed load of shallow waters and their changes. Ocean Engineering, $\mathrm{n}^{\circ} 371$, pp 1174-1198. doi:10.1016/j.oceaneng.2004.12.005

LYZENGA D.R. (1978). Passive remote sensing techniques for mapping water depth and bottom features. Applied Optics, Vol. 17(3), pp 379-383.

TE STROET C., SNEPVANGERS J. (2005). Mapping curvilinear structures with local anisotropy kriging. Math. Geology, Vol. 37(6), pp 635-649. doi:10.1007/s11004-005-7310-y 